Published in final edited form as:

J Am Chem Soc. 2016 September 28; 138(38): 12494-12501. doi:10.1021/jacs.6b06321.

\title{
Using an RNAi Signature Assay To Guide the Design of Three- Drug-Conjugated Nanoparticles with Validated Mechanisms, In Vivo Efficacy, and Low Toxicity
}

\author{
Jonathan C. Barnes ${ }^{\dagger}, \S$, Peter M. Bruno ${ }^{\ddagger}$, , Hung V.-T. Nguyen ${ }^{\dagger}$, Longyan Liao ${ }^{\dagger}$, Jenny Liu ${ }^{\dagger}$, \\ Michael T. Hemann ${ }^{\ddagger}$, and Jeremiah A. Johnson ${ }^{*}, \dagger$ \\ tDepartment of Chemistry, Massachusetts Institute of Technology, 77 Massachusetts Avenue, \\ Cambridge, Massachusetts 02139, United States \\ ¥The David H. Koch Institute for Integrative Cancer Research, Massachusetts Institute of \\ Technology, 77 Massachusetts Avenue, Cambridge, Massachusetts 02139, United States
}

\begin{abstract}
Single-nanoparticle (NP) combination chemotherapeutics are quickly emerging as attractive alternatives to traditional chemotherapy due to their ability to increase drug solubility, reduce offtarget toxicity, enhance blood circulation lifetime, and increase the amount of drug delivered to tumors. In the case of NP-bound drugs, that is, NP-prodrugs, the current standard of practice is to assume that the subcellular mechanism of action for each drug released from the NP mirrors that of the unbound, free-drug. Here, we use an RNAi signature assay for the first time to examine the mechanism of action of multidrug-conjugated NP prodrugs relative to their small molecule prodrugs and native drug mechanisms of action. Additionally, the effective additive contribution of three different drugs in a single-NP platform is characterized. The results indicate that some platinum(IV) cisplatin prodrugs, although cytotoxic, may not have the expected mechanism of action for cisplatin. This insight was utilized to develop a novel platinum(IV) oxaliplatin prodrug and incorporate it into a three-drug-conjugated NP, where each drug's mechanism of action is preserved, to treat tumor-bearing mice with otherwise lethal levels of chemotherapy.
\end{abstract}

\section{Graphical abstract}

*Corresponding Author: jaj2109@mit.edu.

\$Author Contributions: J.C.B. and P.M.B. contributed equally to this work.

Supporting Information

The Supporting Information is available free of charge on the ACS Publications website at DOI: 10.1021/jacs.6b06321.

Synthesis and characterization data for each reported BASP, as well as supplementary figures, methods and materials, procedures, details of signature assays, and additional in vivo data (PDF)

Notes

The authors declare no competing financial interest. 


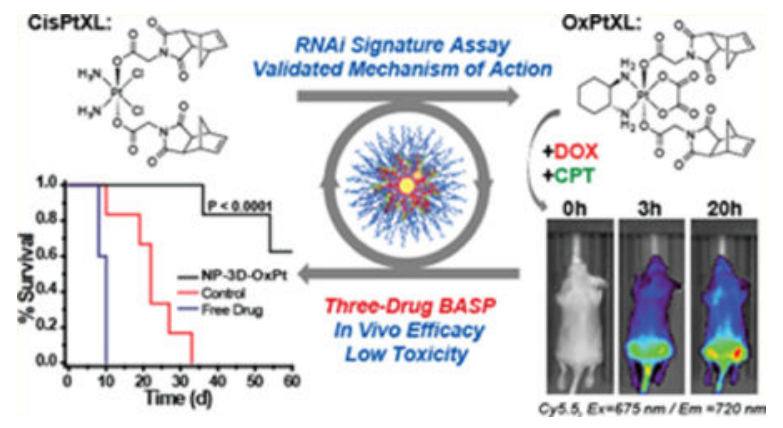

\section{INTRODUCTION}

Traditional cancer chemotherapy is often complicated by the emergence of resistance and/or significant toxicity that can have adverse health ramifications even decades after exposure. ${ }^{1}$ These side effects are often the limiting factor in how much chemotherapy an individual patient can receive, and thus they directly affect patient outcome. Although there are established clinical protocols to ameliorate side effects from specific drug regimens, ${ }^{2}$ new treatment modalities, such as NP-based drug carriers, can increase the quantity of drug a patient receives while reducing negative side effects. ${ }^{3}$ To date, approximately a dozen singledrug-carrying NPs have been approved by the FDA $;^{3}$ however, given that clinical chemotherapy often involves administration of multiple drugs in combination, it stands to reason that the simultaneous delivery of multiple drugs from a single NP platform would allow for better anticipation of drug-drug interactions, and potentially take advantage of synthetic lethal combinations or time-dependent drug synergy. ${ }^{2,4}$ The recent clinical success $^{5}$ of CPX-351, a two-drug carrying liposomal formulation for treatment of acute myeloid lymphoma, provides evidence that multidrug carrying NPs can indeed provide improved patient outcomes, and motivates new studies focused on the design of multidrugcontaining NPs. Indeed, there has been an explosion of interest in the design of multidrug containing NPs for cancer therapy. ${ }^{6}$

Our group has developed ${ }^{7}$ the brush-first ring-opening metathesis polymerization (ROMP) strategy, which has enabled the efficient synthesis of brush-arm star polymer (BASP) NPs of well-defined size that can carry precise ratios of two, three, or potentially more drugs with differing mechanisms of release and action. ${ }^{8}$ In our report on three-drug-conjugated BASPs, ${ }^{8 \mathrm{a}}$ we designed a bis-norbornene cisplatin(IV) diester prodrug that served as the BASP core, a design that was inspired by analogous Pt(IV)-esters and diesters that are widely used as prodrugs and in NP-based Pt drug delivery. ${ }^{9}$ With this system, we demonstrated that NPs with three drugs: Pt(IV)-diester cores and doxorubicin (DOX) and camptothecin (CPT) components, were significantly more cytotoxic in vitro than any combination of two- and one-drug NPs.

Following these studies, we sought a deeper understanding of the subcellular mechanism of action of our BASP NPs. Typically, new drug-carrying NPs are tested on their ability to induce apoptosis or inhibit cell growth in vitro or in vivo, and these observations of cytotoxicity and/or efficacy are assumed to arise from drug release from the NP and then binding of the released drug to the same subcellular target(s) as the corresponding free drug. 
Herein, in an effort to prove that our three-drug-conjugated BASP NPs released their three drugs, and that these drugs displayed the expected additive combination of mechanisms, we applied a simple yet powerful in vitro RNAi signature assay. ${ }^{10}$ Although such assays have been employed to characterize nearly all classes of clinically used anticancer drugs, novel heavy metal-based agents, and combinations of chemotherapeutics, ${ }^{10,11}$ they have not been used to elucidate the mechanism of action of drugs in a NP scaffold.

We were surprised to find that although our drug-conjugated BASP NPs are indeed cytotoxic, and that two of the three NP-bound drugs, DOX and CPT, displayed their expected mechanisms of action as topoisomerase inhibitors, the mechanism of action of the cisplatin prodrug component was quite different from that of free cisplatin. The insight gained from this discovery allowed us to design a new $\mathrm{Pt}(\mathrm{IV})$-prodrug based on oxaliplatin, and a corresponding three-drug-containing BASP NP with validated mechanisms for all three drugs. These particles displayed in vivo efficacy and much lower toxicity as compared to the same three free drugs. We believe that our findings should motivate wide adoption of the RNAi signature assay in NP drug delivery research and design, as it is preferable to translate NP-prodrugs with validated subcellular mechanisms of action to preclinical and clinical studies.

\section{RESULTS AND DISCUSSION}

\section{Nanoparticle Design, Synthesis, and Characterization}

Our NP synthesis is achieved through the design (Figure 1a) of drug-conjugated branched macromonomer $^{12}(\mathrm{MM})$ prodrugs of doxorubicin (DOX) (DOX-MM and its photocleavable (PC) analogue DOX-PC-MM), camptothecin (CPT) (CPT-MM), and platinum(IV) diester cross-linker (XL) prodrugs of cisplatin and oxaliplatin (CisPtXL and OxPtXL, respectively). CisPtXL is the same cross-linker we used previously, ${ }^{8 a}$ whereas $\mathbf{O x P t X L}$ was designed on the basis of insights obtained from the RNAi signature assay (vide infra). See the Supporting Information for the synthesis and characterization of all compounds. Additionally, nondrug-conjugated MMs and XLs were prepared: a $3 \mathrm{kDa}$ poly(ethylene glycol) (PEG)-MM (PEG-MM) ${ }^{7 \mathrm{a}}$ and an acid-sensitive acetal-based cross-linker (AcetalXL), ${ }^{8 b}$ respectively. Each of these MMs and XLs (Figure 1a, Figure S1, and Scheme $\mathrm{S} 1$ ) is functionalized with norbornene units to enable brush-first ROMP, ${ }^{7 \mathrm{a}}$ where the desired MMs are polymerized by exposure to bispyridyl-modified third generation Grubbs initiator $^{7 \mathrm{~b}, 13}(\mathbf{G 3})$ (Figure $1 \mathrm{~b}$ and Scheme S2), which generates bottlebrush polymers comprised of defined ratios of the branched MM drug conjugates and PEG-MM. These living bottlebrush polymers are then cross-linked by addition of AcetalXL, CisPtXL, or OxPtXL, which creates BASP NPs. A key advantage of this brush-first ROMP approach is the ability to swap MMs and/or XLs, which allows for the synthesis of BASPs from any single drug or drug combination that suits a particular treatment regimen with excellent control (>95\% conversion, Figure S6) over relative drug loading ratios and high reproducibility and yield. Using this methodology, we synthesized the series of zero-, one-, and three-drug-conjugated BASPs listed in Figure 1c. Note: We refer to BASPs as "NP" with the corresponding drug MMs and XLs used listed in the name. For example, NP-DOX is a BASP that only carries DOX, which is incorporated via the use of DOX-MM. The name 
"3D" means that the BASP has three drugs. For example, NP-3D-CisPt is prepared from DOX-MM, CPT-MM, and CisPtXL. NP-3D-DD-OxPt is a BASP with three drugs from DOX-MM, CPT-MM, and OxPtXL. "DD" in this case stands for "diluted DOX", and refers to the amount of DOX-MM used in this BASP. NP-3D-PC-OxPt also has three drugs, but uses photocleavable (PC) MM DOX-PC-MM instead of DOX-MM. The relative drug equivalents used in the synthesis and size distributions as determined by dynamic light scattering (DLS) and transmission electron microscopy (TEM) for each BASP are listed in Figure 1c and Table S1. The total theoretical mass fractions of each drug in each BASP are listed in Table S1. For a full description of the synthetic protocols and basic characterization of each BASP reported in this work, see the Supporting Information.

The prodrugs (Figure 1a) reported herein are designed to release their respective native drug in response to various internal or external triggers. Degradation of the core of BASPs derived from platinum XLs is achieved by the reduction of the Pt(IV) diester XL prodrug (i.e., CisPtXL or OxPtXL) to yield, putatively, the cisplatin(II) or oxaliplatin(II) free drugs. ${ }^{14}$ This process leads to release of the BASP's constituent drug-conjugated bottlebrush polymers. Release of DOX and CPT from the nonphotocleavable BASPs (NP-CPT, NPDOX, NP-3D-CisPt, and NP-3D-OxPt) occurs via enzymatic hydrolysis, ${ }^{8 b}, 15$ whereas release of DOX from the photocleavable BASPs (such as NP-3D-PC-OxPt) occurs rapidly upon exposure of the nitrobenzene-functionalized tether to long-wavelength ultraviolet (UV) light. ${ }^{12 a, 16}$ This particle was designed to explicitly test the impact of externally triggered release of DOX on the expected cell killing mechanism; the use of light alone has a negligible adverse effect on cell viability (see the Supporting Information).

\section{RNAi Signature Assay for Studying the Mechanism of Action of Prodrugs and Single-Drug NPs}

As described above, the goal of this investigation is to study the mechanism of action of our BASP NP combination therapies in vitro using an RNAi signature assay. The RNAi signature approach uses murine lymphoma cells that are partially infected with eight different GFP-tagged shRNAs targeting genes related to p53 activation and cell death. ${ }^{10}$ Partial populations of shRNA-bearing cells are dosed (Table S2) with drug such that 80$90 \%$ of the cells are killed $\left(\mathrm{LD}_{80-90}\right)$ at $48 \mathrm{~h}$ to eliminate potential artifacts due to drug metabolism, efflux, etc. Thus, the method involves detailed cytotoxicity studies, but goes further by allowing us to compare the mechanism of cell death to those of classes of known drugs. Together, the relative enrichment or depletion of the eight individual shRNAs in response to drug at $\mathrm{LD}_{80-90}$ is referred to as our "signature". Because the hairpins are targeting genes involved in cell proliferation and survival, this signature is unique for each distinct drug mechanism of action. Thus, we have been able to create a reference set with signatures of drugs with known mechanism of action representing nearly all classes of clinically used cytotoxic drugs, as well as newer, targeted agents. ${ }^{10,11} \mathrm{We}$ are then able to compare the signature of a new drug against this reference set using a modified K-nearest neighbors (K-NN) algorithm. First, K-NN determines which category in the reference set most closely resembles the new drug in question. The new drug is assigned a linkage ratio (LR), which is the pairwise distance of the category member drugs with the new drug included divided by the pairwise distance of the category member drugs without the new 
drug. Thus, a LR $>1$ means the new drug would expand the closest category, and a LR $<1$ means the new drug makes the category smaller. However, if the new drug does not contract the category pairwise distances, a determination must be made of how much expansion is acceptable. To do this, LRs are generated for each drug in the reference set not belonging to the closest category generating a negative control distribution. The linkage ratio of the new drug is compared to the negative control distribution to obtain a $p$-value. If $p<0.05$, then one can confidently classify the new drug as belonging to the closest category. If $p>0.05$, then the drug is determined to exhibit a mechanism of action not represented in the reference set.

Using the RNAi signature assay, we characterized the free drug, prodrug, and, for the first time in NP characterization, the single- and multidrug conjugated BASPs that are illustrated in Figure 1a. It should be noted that all of the drugs, small molecule prodrugs, and BASPs displayed similar levels of cytotoxicity. Thus, on the basis of cytotoxicity alone, we might assume that all of the compounds display their expected mechanism of action. Indeed, the signature assay of native DOX and prodrugs DOX-MM and NP-DOX classified (Figure 2a,e) each species as a Topoisomerase II (Top2) poison. Furthermore, CPT, CPT-MM, and NP-CPT were all classified (Figure 2b,e) as Topoisomerase I (Top1) poisons. As an additional validation of the RNAi signature results, DOX and CPT free drug, as well as NPDOX and NP-CPT, were also tested with validated Top1 and Top2A shRNAs via GFP competition assays (see Supporting Information). Knockdown of Top1 elicited resistance to the Top1 poison CPT and sensitivity to the Top2 poison DOX. The inverse was true for knockdown of Top2A; cells harboring the Top2A shRNA were resistant to free DOX and exhibited sensitivity to free CPT. ${ }^{17}$ These results were recapitulated for the respective BASPs, specifically NP-CPT and NP-DOX, where each BASP produced (Figure 2f) responses similar to those of their corresponding free drugs CPT and DOX, respectively.

Although our DOX and CPT prodrug NPs displayed the expected mechanisms of action, the prodrugs CisPtXL and NP-CisPt classified (Figure 2c,e) as new categories not represented in the reference set, and not as DNA cross-linkers, the anticipated mechanism of action of free cisplatin. Thus, our cisplatin prodrug has an unexpected subcellular mechanism of action despite its cytotoxicity. To investigate the mechanisms of these compounds more deeply, the RNAi signatures for each of the free drugs and their corresponding prodrug monomers and BASPs were plotted (Figure 2e) using principal component analysis (PCA). PCA is a means of representing the variance in a multidimensional data set in fewer dimensions. These data indicate that the CisPtXL and NP-CisPt are both more similar to the transcription/translation inhibitors than to the DNA cross-linkers. To support this observation, we used a CisPt-DNA adduct specific antibody ${ }^{18}$ to analyze genomic DNA isolated from murine lymphoma cells after $8 \mathrm{~h}$ of free cisplatin versus CisPtXL treatment. Cisplatin-DNA adducts were only detected (Figure S7) as a result of cisplatin (CisPt) treatment, and not CisPtXL, further confirming that the latter is not acting as a DNA crosslinker.

One hypothesis that may explain this unexpected mechanism is that our cisplatin prodrugs are not forming free cisplatin upon intracellular reduction, and that they are forming an alternative, yet still cytotoxic platinum species. Indeed, the reduction of $\mathrm{Pt}(\mathrm{IV})$ prodrugs has 
been observed to yield various $\mathrm{Pt}(\mathrm{II})$ products based on the reaction conditions and the reducing agent used. ${ }^{14 c, 19} \mathrm{On}$ the basis of analogy to these studies, it is possible that an axial norbornene-carboxylate ligand in our cisplatin prodrugs shifts to an equatorial position of the platinum center, thus forming a carboxylate-Pt(II) complex rather than cisplatin. On the basis of our data, this new complex is still cytotoxic, but it displays a significantly different pattern of DNA binding than cisplatin, potentially forming a majority of monofunctional DNA adducts.

With this hypothesis in mind, we designed oxaliplatin (OxPt) prodrug OxPtXL (Figure 1a). Oxaliplatin is a clinically used agent for colorectal cancer, and although it is also a Pt(II) agent, its spectrum of activity is quite distinct from that of cisplatin, and it tends to be effective even against cisplatin-resistant cell lines. ${ }^{20}$ Notably, oxaliplatin classifies as a transcription/translation inhibitor and not a DNA cross-linker in our RNAi signatures assay. Thus, even if the less labile oxalate ligands of OxPtXL were displaced by norbornene carboxylate ligands, the expected mechanism of action would not be changed. Indeed, both the free drug OxPt and the NP-OxPt classified as transcription/translation inhibitors (Figure 2d). Interestingly, the prodrug crossinker OxPtXL did not significantly classify as a transcription/translation inhibitor, but transcription/translation inhibition was the most similar category. Furthermore, our molecular rationale for the mechanistic differences between NP-CisPt and NP-OxPt, which is based on binding of a norbornene carboxylate ligand to an equatorial position of the Pt center, is supported by a comparison of the corresponding Pt(II) release profiles (Figure S8) associated with each NP in the presence of glutathione (GSH) reducing agent. Whereas release of Pt from NP-OxPt plateaus at approximately 75 days, release of platinum from NP-CisPt never plateaus within the scope of a 270-day investigation, suggesting that platinum remains bound to the polymeric carrier. Given these observations, we selected OxPtXL rather than CisPtXL for use in all of the subsequent studies. These results show how our simple RNAi signature assay can help guide the design of NPs with validated subcellular mechanisms of action.

\section{Using RNAi Signatures and Constrained Linear Regression To Determine the Effective Contributions of Drugs in a Three-Drug-Conjugated BASP}

To use RNAi signatures to determine the relative effective contribution of multiple drugs with additive toxicities within a single NP, we first conducted a Bliss Independence ${ }^{21}$ analysis (Figure S9) to confirm that the three drugs used in this investigation display additive toxicity rather than synergistic or antagonistic toxicity. Given an additive drug combination, it should be possible to extract the contribution of each drug to the cell killing mechanism using constrained linear regression methods (if a synergistic drug combination were used, the RNAi signature assay would be dominated by the signature of a single drug in the combination). ${ }^{11 \mathrm{~b}}$

A BASP capable of externally triggered drug release was designed wherein we could easily validate the presence or absence of the triggered drug in a combination of other drugs without having to change the BASP composition. For this, CPT-MM and photocleavable DOX-PC-MM were cross-linked with OxPtXL via brush-first ROMP to yield three-drugconjugated BASP NP-3D-PC-OxPt. The incorporation of the UV-triggered DOX-PC-MM 
allows for rapid release of free DOX upon exposure to $365 \mathrm{~nm}$ light. ${ }^{16 \mathrm{~b}}$ NP-3D-PC-OxPt was used to treat cells either with or without long wavelength light irradiation ( $\triangleleft \mathrm{min}$; this dose of light has a negligible impact on cell viability; Figure S10), and RNAi signatures were obtained. Constrained linear regression was then performed (Figure 3a and Figure S10a,b) on the RNAi signatures to ascertain the relative effective contributions of each drug's mechanism of action between the UV-irradiated and non-irradiated BASP treatments. As anticipated, the signatures obtained in the absence of light irradiation showed (Figure 3a, "no UV") that free DOX was not predicted to have contributed to the mechanism of action of the NP, as determined by constrained linear regression. Conversely, constrained linear regression predicted (Figure 3a, "UV") that free DOX contributed to the UV-irradiated NP mechanism of action at approximately $55 \%$. We then analyzed the mechanism of action of two novel BASPs that either contained DOX-PC-MM diluted down to $\sim 0.2 \%$ of the amount used in NP-3D-PC-OxPt (the so-called NP-3D-DD-OxPt, where DD = diluted DOX), or possessed the non-photocleavable DOX-MM (thus, NP-3D-OxPt). In both of these cases, regardless of treatment with UV light, constrained linear regression was able to identify (Figure 3b,c and Figure S10a,b) the contribution, or lack thereof in the case of the diluted DOX NP, of free DOX to the combined mechanism of action.

With the ability to discern individual drug contributions via constrained linear regression, NP-3D-OxPt was analyzed in more detail in terms of the quantitative percent contribution that the CPT, DOX, and OxPt components, all drugs whose individual NPs were determined to recapitulate their corresponding native behavior, exhibit toward the overall mechanism of action. Upon testing the combination via RNAi signature and constrained linear regression, it was determined (Figure 3c and Figure S10a,b) that free CPT, DOX, and OxPt contributed approximately $38 \%, 26 \%$, and $36 \%$, respectively, toward the killing of cancer cells.

The studies described above demonstrate that with our simple RNAi signature assay it is possible to validate the subcellular mechanisms of action and contributions to cell killing of multiple drugs in a single nanoparticle for the first time. With this assay as a guiding tool, we identified NP-3D-OxPt as an ideal particle for translation to in vivo studies. It should be noted that because the subcellular mechanism of a chemotherapeutic agent is usually indifferent to the cell type, for example, DOX is a Topo II inhibitor regardless of the cell type, the mechanism of action as determined by our RNAi signature assay in lymphoma cells can be reasonably expected to translate to other cells. In fact, we view this translatability across cell types as a major advantage of the assay, which has been demonstrated in non-NP systems. ${ }^{11 \mathrm{~b}}$ Furthermore, in vitro RNAi signature results have been recapitulated precisely in vivo. ${ }^{22}$ The CPT, DOX, OxPt combination in NP-3D-OxPt is relevant for potential applications in ovarian cancer therapy where platinum agents and topoisomerase inhibitors are current first- and second-line therapies; thus, in the studies described below, we focus on in vivo efficacy of this particle in an ovarian cancer tumor model. 


\section{In Vivo Efficacy Studies of Three-Drug-Conjugated BASP with Validated Subcellular Mechanism of Action}

To investigate the therapeutic efficacy of the three-drug-conjugated NP-3D-OxPt in vivo, NCR-NU mice were injected subcutaneously in each hind flank with $1.25 \times 10^{6}$ ovarian carcinoma cells (SKOV-3, ATCC) mixed (1:1) with Matrigel and PBS buffer. Tumor growth was monitored for 2-4 wks until the tumor reached approximately $0.5 \mathrm{~cm}$ in diameter, at which point three treatment groups were established: those treated with sterile-filtered 5\% aqueous glucose solutions containing either (i) NP-3D-OxPt, (ii) a free drug formulation at the same DOX, CPT, and OxPt concentrations as NP-3D-OxPt, or (iii) the blank vehicle. The BASP treatment schedule consisted of four tail-vein injections over $22 \mathrm{~d}(\sim 1 \mathrm{inj} . / \mathrm{wk})$, where each injection consisted of $5 \mathrm{mg}$ of BASP in $200 \mu \mathrm{L}$ of $5 \%$ glucose solution, a dose that is close to the maximum solubility limit ( $\sim \mathrm{mg} / 200 \mu \mathrm{L}$ ) of the three-drug-conjugated BASP. Because $\sim 10 \%$ of each NP-3D-OxPt is made up of the three anticancer drugs, each dose is equal to $\sim 20 \mathrm{mg}$ total drug/kg mouse (Table S1). Simultaneous delivery of all three drugs within one BASP entity ensures that the three drugs will arrive at the tumor in a ratio defined by the BASP, which precludes differences in pharmacokinetics and biodistribution that could be observed for mixtures of single-drug-conjugated NPs. Therefore, subcellular mechanistic results obtained in cell culture can be expected to translate to the tumor.

As a consequence of the branched MM design, the BASP possesses a PEG shell that may provide for enhanced in vivo circulation time. ${ }^{23}$ Indeed, we observed a blood circulation half-life $\left(t_{1 / 2}\right)$ of $3.2 \mathrm{~h}$ for NP-3D-OxPt with $1 \%$ Cy5.5-MM ${ }^{24}$ in NCR-NU mice (Figure S11a), which allows sufficient time for passive accumulation of the BASPs in subcutaneous tumors (Figure S12a,b for NP-OxPt conjugated with 1\% Cy5.5-MM, and Figure 4a for NP-3D-OxPt conjugated with 1\% Cy5.5-MM) within 20-24 h postinjection via the enhanced permeability and retention (EPR) effect. ${ }^{25}$ A qualitative biodistribution analysis of NP-3D-OxPt with 1\% Cy5.5-MM in BALB/c mice (Figure S11b) and NP-OxPt conjugated with 1\% Cy5.5-MM in tumor-laden NCR-NU mice (Figure S12b,c) reveals liver and tumor accumulation after $24 \mathrm{~h}$, respectively, followed by excretion of the residual NP in the mouse feces after $48 \mathrm{~h}$.

The mice treated with the free drug formulation lost significant total body mass after only two treatments (Figure $4 \mathrm{~b}$, blue trace, $>10 \%$ average reduction) and therefore had to be euthanized, with their blood serum and tissues harvested for post-therapeutic analysis. The BASP treatment group, however, displayed (Figure 4b; black trace, BASP; red trace, vehicle) excellent therapeutic tolerance, as evidenced by a consistent total body mass and no noticeable adverse effects throughout the course of the study. These results validate the ability of the BASP NP to deliver each drug at or above its MTD in comparison to the corresponding free drug cocktail. The tumor volume progression plot illustrates (Figure 4c) a clear regression in tumor growth associated with the BASP treatment group, whereas the mice in the vehicle control group showed no signs of tumor regression. After the fourth and final BASP treatment, both the BASP treatment and the vehicle groups showed a continued increase of tumor volumes, although the tumors in the former case grew at a slower rate. The survival rate of each treatment group reflects (Figure 4d) the overall therapeutic tolerance and efficacy associated with the BASP combination therapy, whereas the mice from the 
vehicle control group were removed as deemed necessary, according to the criteria established in the MIT Committee on Animal Care protocol. After completion of the $60 \mathrm{~d}$ therapeutic efficacy study, the surviving mice (referred to as "chronic mice") from the BASP treatment group were sacrificed, and their blood serum and organs were harvested to perform a blood panel analysis (Figure S13). This study allowed for comparison of toxicity between the poststudy chronically treated mice, the midstudy acutely treated mice, and vehicle mice. As anticipated, the acutely and chronically treated mice associated with the BASP treatment group demonstrated little to no kidney damage as evidenced by the low blood urea nitrogen to creatinine ratio (B/C; Figure S13), as well as very low amounts of liver damage-related biomarkers, such as alanine and aspartate aminotransferases (ALT and AST, respectively, Figure S13). Pathology of the paraffin-embedded, H\&E-stained cross sections ( $\sim 5 \mathrm{~m}$ thick) of the livers (Figure S14) from the BASP-treated and vehicle mice supports this biomarker quantification analysis. Cross sections of the paraffin-embedded, H\&E-stained tumors harvested from acute/chronic BASP-treated mice show (Figure S15) strong evidence of cancer cell death in direct contrast to the healthy vehicle control tumor tissue. To assess the degree to which our NP-3D-OxPt dose could demonstrate efficacy in larger tumors from the same cancer cell line, we carried out another in vivo therapeutic efficacy study (Figure S16) starting with tumors $\sim 1.0 \mathrm{~cm}$ in diameter and observed comparable therapeutic tolerance and efficacy relative to the results from the first study.

\section{CONCLUSIONS}

We have demonstrated for the first time that RNAi signature assays can be used to design and validate the subcellular mechanisms of NP combination therapies for cancer. With this tool, we prepared a novel BASP NP comprised of three drugs that displayed the expected mechanisms of action and nearly equal contributions to cell killing for all three drugs. We then showed that this ROMP-based BASP NP is an effective construct for delivering a precise ratio of an otherwise toxic combination of three drugs to a subcutaneous xenograft tumor in mice. To our knowledge, this study is the first report of using ROMP-based NPs for in vivo combination cancer therapy. This work highlights the fact that the observation of cytotoxicity from a NP-bound drug does not necessarily mean that the drug is operating via its expected mechanism of action, as we observed for our cisplatin constructs. Instead, the mechanism of action should be validated regardless of observed efficacy or cytotoxicity. The RNAi signature assay described here allows for a fast and accurate in vitro combinatorial screening that is capable of predicting a prodrug NP's mechanism of action. Although our $\mathrm{Pt}(\mathrm{IV})$-diester XL, intended to be a precursor to cisplatin, did not behave as anticipated in vitro, the RNAi signatures led us to develop a novel Pt(IV)-diester XL that functions as a faithful precursor to OxPt. Moreover, constrained linear regression analysis, paired with RNAi signatures, revealed the relative effective contributions of each drug toward the mechanism of action in our three-drug-conjugated BASPs. The ability to assess the contribution in vitro of each drug adds another layer of quality control to NP-based drug delivery, which should translate to in vivo applications because subcellular mechanisms of action are not expected to change. The initial stoichiometric ratios can be tuned to achieve BASPs where all drugs contribute equally, or BASPs that possess disparate drug contributions that may potentially maximize each drug's MTD, all the while taking into 
account the kinetics associated with the release of each drug from the combination NP platform. Looking ahead, this modular platform and the RNAi-based mechanism of action predictive assay can be further utilized to assess different combinations of drugs in BASPs in an effort to treat other types of cancer. We expect that the RNAi signature assay can be widely employed to characterize other types of nanoparticles different from BASPs that also carry one, two, three, or potentially more drugs.

\section{Supplementary Material}

Refer to Web version on PubMed Central for supplementary material.

\section{Acknowledgments}

J.C.B. was supported by the Howard Hughes Medical Institute as a Postdoctoral Fellow of the Life Sciences Research Foundation. P.M.B. is supported by the Integrative Cancer Biology Program and the Ludwig Institute. H.V.-T.N. is supported by the National Science Foundation as a Graduate Research Fellow. J.A.J. thanks the National Institutes of Health NIBIB (1R21EB018529-01A1) for support. We thank Dr. Erik C. Dreaden for very fruitful discussions and consultation in relation to the in vivo therapeutic efficacy studies.

\section{References}

1. (a) Schwartz CL. Oncologist. 1999; 4:45. [PubMed: 10337370] (b) Luqmani YA. Med Prin Pract. 2005; 14:35.(c) Mellor HR, Callaghan R. Pharmacology. 2008; 81:275. [PubMed: 18259091] (d) Diaz LA, Williams RT, Wu J, Kinde I, Hecht JR, Berlin J, Allen B, Bozic I, Reiter JG, Nowak MA, Kinzler KW, Oliner KS, Vogelstein B. Nature. 2012; 486:537. [PubMed: 22722843] (e) Zaharov T, Wehner PS, Schomerus E, Bech ESN. Pediatr Blood Cancer. 2013; 60:162.

2. McLornan DP, List A, Mufti GJ. N Engl J Med. 2014; 371:1725. [PubMed: 25354106]

3. Wicki A, Witzigmann D, Balasubramanian V, Huwyler J. J Controlled Release. 2015; 200:138.

4. Lee MJ, Ye AS, Gardino AK, Heijink AM, Sorger PK, MacBeath G, Yaffe MB. Cell. 2012; 149:780. [PubMed: 22579283]

5. (a) Lancet JE, Cortes JE, Hogge DE, Tallman MS, Kovacsovics TJ, Damon LE, Komrokji R, Solomon SR, Kolitz JE, Cooper M, Yeager AM, Louie AC, Feldman EJ. Blood. 2014; 123:3239. [PubMed: 24687088] (b) Lancet JE, Uy GL, Cortes JE, Newell LF, Lin TL, Ritchie EK, Stuart RK, Strickland SA, Hogge D, Solomon SR, Stone RM, Bixby DL, Kolitz JE, Schiller GJ, Wieduwilt MJ, Ryan DH, Hoering A, Chiarella M, Louie AC, Medeiros BC. J Clin Oncol. 2016:34.

6. (a) Hu CMJ, Zhang L. Biochem Pharmacol. 2012; 83:1104. [PubMed: 22285912] (b) Ma L, Kohli M, Smith A. ACS Nano. 2013; 7:9518. [PubMed: 24274814] (c) Meng H, Mai WX, Zhang HY, Xue M, Xia T, Lin SJ, Wang X, Zhao Y, Ji ZX, Zink JI, Nel AE. ACS Nano. 2013; 7:994. [PubMed: 23289892] (d) Deng ZJ, Morton SW, Ben-Akiva E, Dreaden EC, Shopsowitz KE, Hammond PT. ACS Nano. 2013; 7:9571. [PubMed: 24144228] (e) Liboiron BD, Mayer LD. Ther Delivery. 2014; 5:149.(f) Mignani S, Bryszewska M, Klajnert-Maculewicz B, Zablocka M, Majoral JP. Biomacromolecules. 2015; 16:1. [PubMed: 25426779] (g) Gadde S. MedChemComm. 2015; 6:1916.(h) Au KM, Min YZ, Tian X, Zhang LZ, Perello V, Caster JM, Wang AZ. ACS Nano. 2015; 9:8976. [PubMed: 26267360] (i) Kinoh H, Miura Y, Chida T, Liu XY, Mizuno K, Fukushima S, Morodomi Y, Nishiyama N, Cabral H, Kataoka K. ACS Nano. 2016; 10:5643. [PubMed: 27093466]

7. (a) Liu J, Burts AO, Li Y, Zhukhovitskiy AV, Ottaviani MF, Turro NJ, Johnson JA. J Am Chem Soc. 2012; 134:16337. [PubMed: 22953714] (b) Liu J, Gao AX, Johnson JA. J Vis Exp. 2013:e50874.

8. (a) Liao L, Liu J, Dreaden EC, Morton SW, Shopsowitz KE, Hammond PT, Johnson JA. J Am Chem Soc. 2014; 136:5896. [PubMed: 24724706] (b) Gao AX, Liao L, Johnson JA. ACS Macro Lett. 2014; 3:854. [PubMed: 25243099]

9. (a) Jamieson ER, Lippard SJ. Chem Rev. 1999; 99:2467. [PubMed: 11749487] (b) Hall MD, Hambley TW. Coord Chem Rev. 2002; 232:49.(c) Nishiyama N, Okazaki S, Cabral H, Miyamoto M, Kato Y, Sugiyama Y, Nishio K, Matsumura Y, Kataoka K. Cancer Res. 2003; 63:8977. [PubMed: 14695216] (d) Jung YW, Lippard SJ. Chem Rev. 2007; 107:1387. [PubMed: 17455916] 
(e) Kolishetti N, Dhar S, Valencia PM, Lin LQ, Karnik R, Lippard SJ, Langer R, Farokhzad OC. Proc Natl Acad Sci U S A. 2010; 107:17939. [PubMed: 20921363] (f) Wilson JJ, Lippard SJ. Inorg Chem. 2011; 50:3103. [PubMed: 21361279] (g) Chin CF, Wong DYQ, Jothibasu R, Ang WH. Curr Top Med Chem. 2011; 11:2602. [PubMed: 22039869] (h) Huynh VT, Binauld S, de Souza PL, Stenzel MH. Chem Mater. 2012; 24:3197.(i) Scarano W, Duong HTT, Lu HX, De Souza PL, Stenzel MH. Biomacromolecules. 2013; 14:962. [PubMed: 23469757] (j) Zheng YR, Suntharalingam K, Johnstone TC, Yoo H, Lin W, Brooks JG, Lippard SJ. J Am Chem Soc. 2014; 136:8790. [PubMed: 24902769] (k) Johnstone TC, Suntharalingam K, Lippard SJ. Chem Rev. 2016; 116:3436. [PubMed: 26865551] (1) Czapar AE, Zheng YR, Riddell IA, Shukla S, Awuah SG, Lippard SJ, Steinmetz NF. ACS Nano. 2016; 10:4119. [PubMed: 26982250]

10. Jiang H, Pritchard JR, Williams RT, Lauffenburger DA, Hemann MT. Nat Chem Biol. 2011; 7:92. [PubMed: 21186347]

11. (a) Pritchard JR, Bruno PM, Hemann MT, Lauffenburger DA. Mol BioSyst. 2013; 9:1604. [PubMed: 23287973] (b) Pritchard JR, Bruno PM, Gilbert LA, Capron KL, Lauffenburger DA, Hemann MT. Proc Natl Acad Sci U S A. 2013; 110:E170. [PubMed: 23251029] (c) Suntharalingam K, Johnstone TC, Bruno PM, Lin W, Hemann MT, Lippard SJ. J Am Chem Soc. 2013; 135:14060. [PubMed: 24041161] (d) Suntharalingam K, Lin W, Johnstone TC, Bruno PM, Zheng YR, Hemann MT, Lippard SJ. J Am Chem Soc. 2014; 136:14413. [PubMed: 25247635] (e) Suntharalingam K, Awuah SG, Bruno PM, Johnstone TC, Wang F, Lin W, Zheng YR, Page JE, Hemann MT, Lippard SJ. J Am Chem Soc. 2015; 137:2967. [PubMed: 25698398] (f) Awuah SG, Zheng YR, Bruno PM, Hemann MT, Lippard SJ. J Am Chem Soc. 2015; 137:14854. [PubMed: 26561720]

12. (a) Johnson JA, Lu YY, Burts AO, Xia Y, Durrell AC, Tirrell DA, Grubbs RH. Macromolecules. 2010; 43:10326. [PubMed: 21532937] (b) Johnson JA, Lu YY, Burts AO, Lim YH, Finn MG, Koberstein JT, Turro NJ, Tirrell DA, Grubbs RH. J Am Chem Soc. 2011; 133:559. [PubMed: 21142161]

13. (a) Sanford MS, Love JA, Grubbs RH. Organometallics. 2001; 20:5314.(b) Love JA, Morgan JP, Trnka TM, Grubbs RH. Angew Chem, Int Ed. 2002; 41:4035.

14. (a) Fuertes MA, Alonso C, Perez JM. Chem Rev. 2003; 103:645. [PubMed: 12630848] (b) Hall MD, Alderden RA, Zhang M, Beale PJ, Cai ZH, Lai B, Stampfl APJ, Hambley TW. J Struct Biol. 2006; 155:38. [PubMed: 16630726] (c) Gibson D. Dalton Trans. 2016; 45:12983. [PubMed: 27214873]

15. (a) Liederer BM, Borchardt RT. J Pharm Sci. 2006; 95:1177. [PubMed: 16639719] (b) Parrish B, Emrick T. Bioconjugate Chem. 2007; 18:263.(c) Parrish B, Emrick T. Bioconjugate Chem. 2007; $18: 263$.

16. (a) Agasti SS, Chompoosor A, You CC, Ghosh P, Kim CK, Rotello VM. J Am Chem Soc. 2009; 131:5728. [PubMed: 19351115] (b) Burts AO, Liao L, Lu YY, Tirrell DA, Johnson JA. Photochem Photobiol. 2014; 90:380. [PubMed: 24117423]

17. Burgess DJ, Doles J, Zender L, Xue W, Ma B, McCombie WR, Hannon GJ, Lowe SW, Hemann MT. Proc Natl Acad Sci U S A. 2008; 105:9053. [PubMed: 18574145]

18. Tilby MJ, Johnson C, Knox RJ, Cordell J, Roberts JJ, Dean CJ. Cancer Res. 1991; 51:123. [PubMed: 1703029]

19. (a) Sinisi M, Intini FP, Natile G. Inorg Chem. 2012; 51:9694. [PubMed: 22921246] (b) Nemirovski A, Vinograd I, Takrouri K, Mijovilovich A, Rompel A, Gibson D. Chem Commun. 2010; 46:1842. (c) Ravera M, Gabano E, Zanellato I, Bonarrigo I, Escribano E, Moreno V, Font-Bardia M, Calvet T, Osella D. Dalton Trans. 2012; 41:3313. [PubMed: 22286213]

20. Apps MG, Choi EHY, Wheate NJ. Endocr-Relat Cancer. 2015; 22:R219. [PubMed: 26113607]

21. Bliss CI. Bacteriol Rev. 1956; 20:243. [PubMed: 13403845]

22. Zhao BY, Pritchard JR, Lauffenburger DA, Hemann MT. Cancer Discovery. 2014; 4:166. [PubMed: 24318931]

23. (a) Moghimi SM, Hunter AC. Crit Rev Ther Drug. 2001; 18:527.(b) Jokerst JV, Lobovkina T, Zare RN, Gambhir SS. Nanomedicine. 2011; 6:715. [PubMed: 21718180]

24. Sowers MA, McCombs JR, Wang Y, Paletta JT, Morton SW, Dreaden EC, Boska MD, Ottaviani MF, Hammond PT, Rajca A, Johnson JA. Nat Commun. 2014; 5:5460. [PubMed: 25403521] 
25. (a) Matsumura Y, Maeda H. Cancer Res. 1986; 46:6387. [PubMed: 2946403] (b) Duncan R. Pharm Sci Technol Today. 1999; 2:441. [PubMed: 10542390] (c) Torchilin V. Adv Drug Delivery Rev. 2011; 63:131.(d) Wilhelm S, Tavares AJ, Dai Q, Ohta S, Audet J, Dvorak HF, Chan WCW. Nat Rev Mater. 2016:1. 

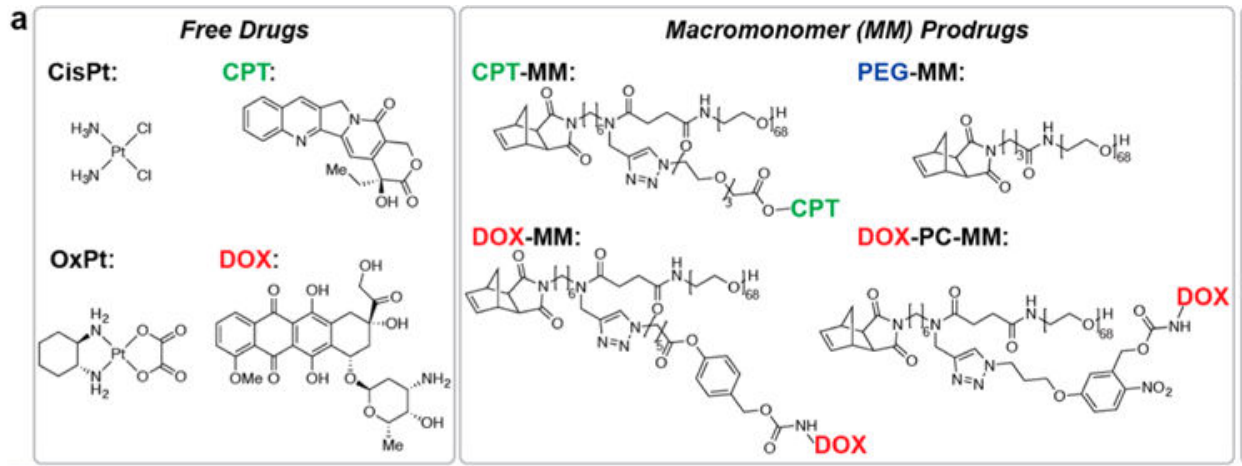

Cross-Linker
Prodrugs $(P t-X L s)$ Prodrugs (Pt-XLs) CisPtXL:
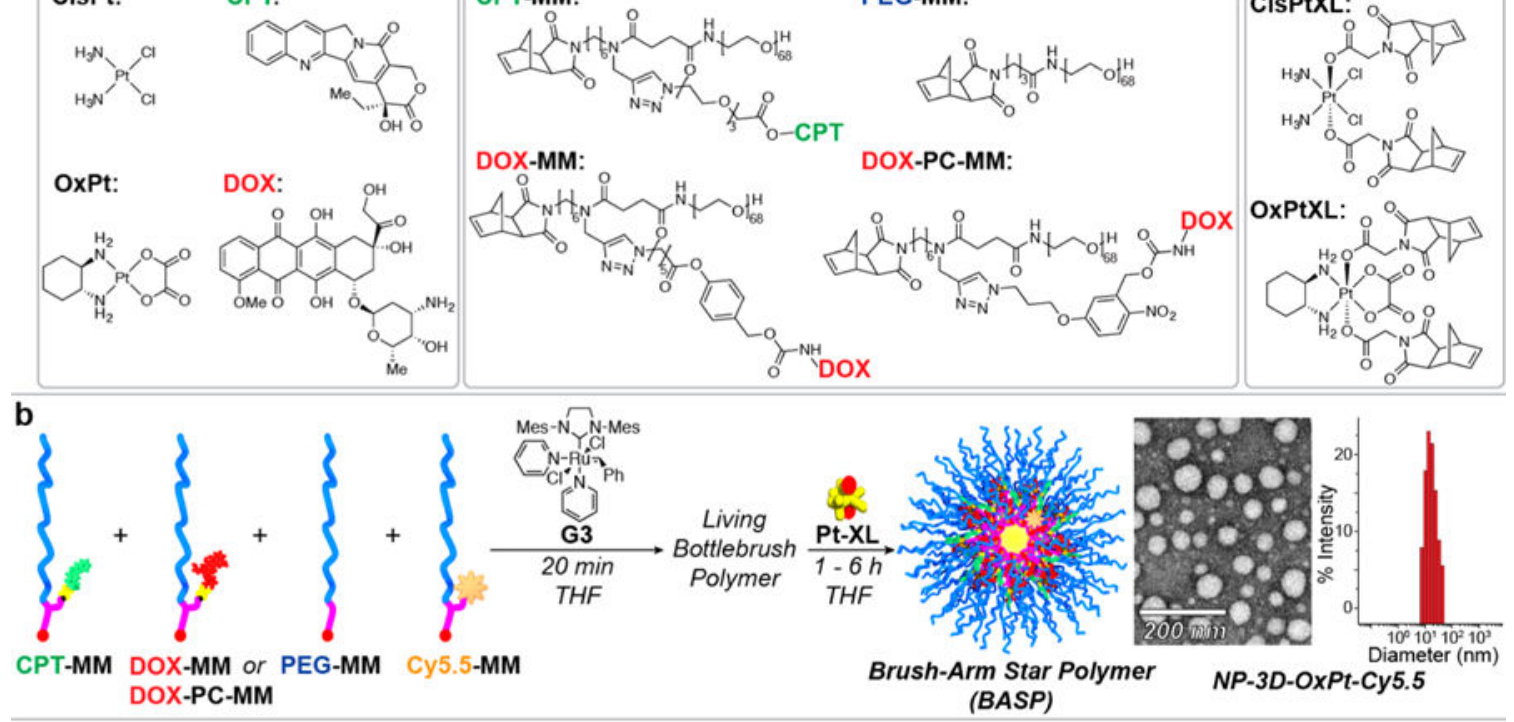

\begin{tabular}{|c|c|c|c|c|c|c|c|c|}
\hline \multicolumn{2}{|l|}{ C } & \multicolumn{5}{|c|}{ Equivalents of $M M$ and $X L$ Relative to G3 } & \multicolumn{2}{|c|}{ BASP Sizes } \\
\hline BASP Nanoparticle & Composition & PEG-MM & СРТ-MM & DOX-MM & DOX-PC-MM & $\mathbf{X L}$ & $D_{\mathrm{H}} \quad(\mathrm{nm})$ & $D_{\text {TEM }}$ \\
\hline NP-PEG & blank & 7.02 & - & - & - & 14.4 & $30 \pm 12$ & $52 \pm 9$ \\
\hline NP-CPT & CPT only & 8.66 & 1.40 & - & - & 15.0 & $64 \pm 41$ & $48 \pm 11$ \\
\hline NP-DOX & DOX only & 8.66 & - & 1.40 & - & 15.0 & $44 \pm 12$ & $39 \pm 9$ \\
\hline NP-OxPt & OxPt only & 10.00 & - & - & - & 5.0 & n. d. & $34 \pm 8$ \\
\hline NP-3D-DD-OxPt & CPT, Diluted DOX, OxPt & 4.99 & 5.00 & - & 0.01 & 5.0 & $37 \pm 21$ & $57 \pm 33$ \\
\hline NP-3D-PC-OxPt & CPT, DOX, OxPt & 5.15 & 3.46 & - & 1.39 & 5.0 & $16 \pm 3$ & n. d. \\
\hline NP-3D-OxPt & CPT, DOX, OxPt & 5.15 & 3.46 & 1.39 & - & 5.0 & $21 \pm 6$ & n. d. \\
\hline NP-3D-CisPt & CPT, DOX, CisPt & 4.11 & 2.08 & 0.84 & - & 3.0 & n. d. & n. d. \\
\hline NP-3D-OxPt-Cy5.5 & CPT, DOX, OxPt, $1 \%$ Cy5.5 & $5 \quad 5.02$ & 3.45 & 1.39 & - & 5.0 & $17 \pm 6$ & $45 \pm 14$ \\
\hline
\end{tabular}

Figure 1.

Chemical structures of free drugs, prodrug branched macromonomers (MMs), and crosslinkers (XLs); a general nanoparticle synthesis; and nanoparticle composition and size distribution data. (a) Chemical structures are provided for the free drugs, MMs, and XLs reported in this investigation. (b) A general reaction scheme illustrates the process of synthesizing drug-conjugated BASP NPs using "brush-first" ROMP. The TEM image (scale bar $=200 \mathrm{~nm}$ ) to the right of the BASP graphic shows one batch of three-drug-conjugated NPs comprised of CPT, DOX, OxPt prodrugs (i.e., MMs and XLs), and 1\% Cy5.5 fluorophore. (c) Data table that lists each BASP NP reported in this work, as well as the prodrug composition and size distribution characterization data. Note: The XL for the first three entries is the AcetalXL. Also, the NP-3D-CisPt size distribution was reported previously; ${ }^{8 \mathrm{a}}$ it should be noted that the particles in this work are generally smaller than those in our previous work as we optimized conditions to avoid aggregation during DLS and TEM analysis. 
a

e

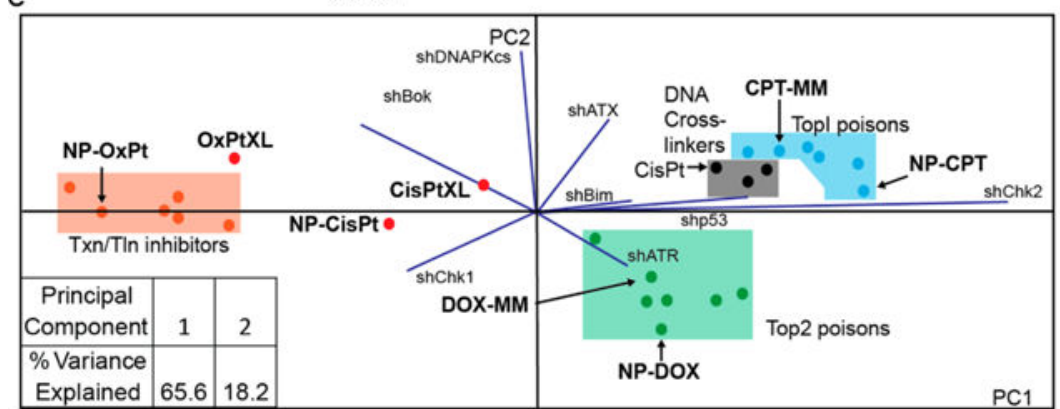

d
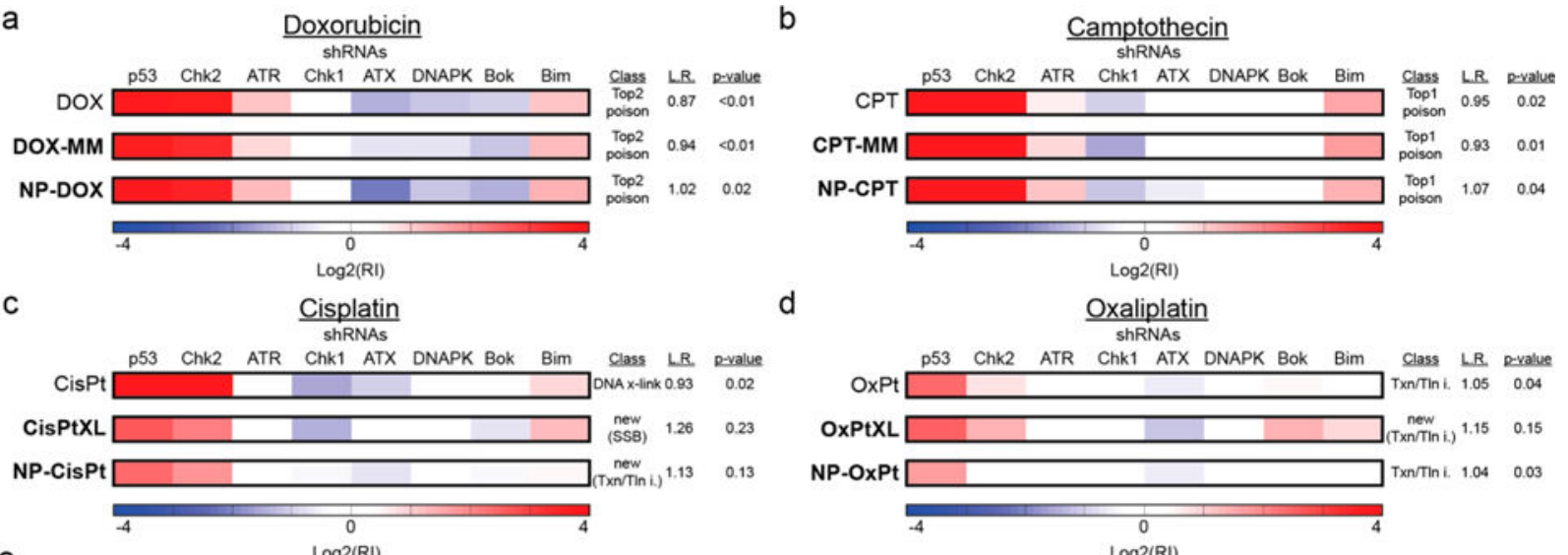

Figure 2.

RNAi signatures and classification of individual free drugs, prodrugs, and nanoparticles. (a)

Heat maps and classification of native DOX, prodrug DOX-MM, and BASP NP-DOX. (b)

Heat maps and classification of native CPT, prodrug CPT-MM, and BASP NP-CPT. (c)

Heat maps and classification of native CisPt, prodrug CisPtXL, and BASP NP-CisPt. (d)

Heat maps and classification of native OxPt, prodrug OxPtXL, and BASP NP-OxPt. (e)

Principal component analysis of RNAi signatures from native, prodrug, and nanoparticles of

DOX, CPT, CisPt, and OxPt, as well as representative drugs from transcription/translation

(Txn/Tln) inhibitor, Topoisomerase II (Top2) poison, Topoisomerase I (Top1) poison, and

DNA cross-linker reference set categories. (f) Heat map depicting response of Top1 and Top2A shRNA-bearing cells to treatment with DOX or CPT free drug or nanoparticles (NP-

DOX and NP-CPT, respectively). 
Doxorubicin $\square$ Camptothecin $\square$ Oxaliplatin

a NP-3D-PC-OxPt (photo-released DOX)

no UV UV
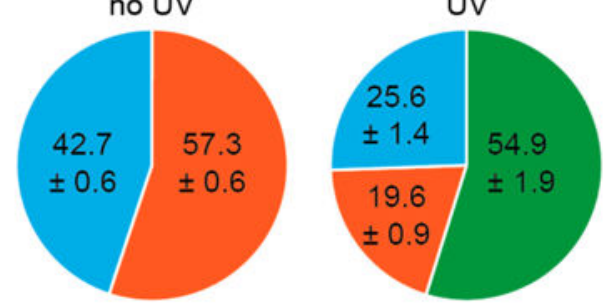

b

NP-3D-DD-OxPt (diluted DOX)

no UV

UV
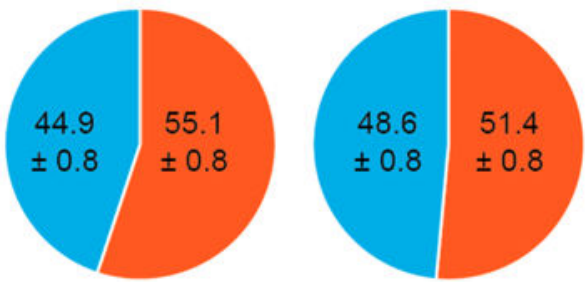

C

NP-3D-OxPt
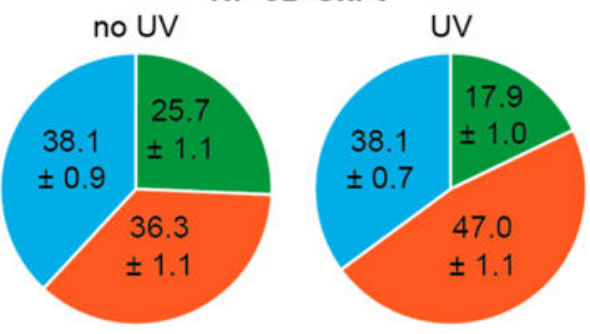

Figure 3.

Contribution of different drugs to overall mechanism of action of three-drug-conjugated nanoparticles as predicted by constrained linear regression. (a) Constrained linear regression prediction of contribution to mechanism of action of a three-drug-conjugated BASP NP (i.e., NP-3D-PC-OxPt) that has photocleavable (PC) DOX MM with or without UV irradiation at $365 \mathrm{~nm}$. (b) Constrained linear regression prediction of contribution of mechanism of action (with or without UV irradiation) of a related, three-drug-conjugated NP that has a very diluted ( 0.2\%) amount of DOX-PC-MM. (c) Constrained linear regression prediction of contribution to mechanism of action (with or without UV irradiation) of a three-drugconjugated NP (i.e., NP-3D-OxPt) that has a non-photocleavable (i.e., ester linker) DOXMM. The reported values represent the mean and standard error of the mean. 
a<smiles></smiles>

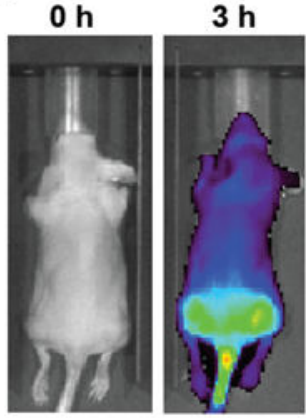

Cy5.5, Ex $=675 \mathrm{~nm} / \mathrm{Em}=720 \mathrm{~nm}$

C

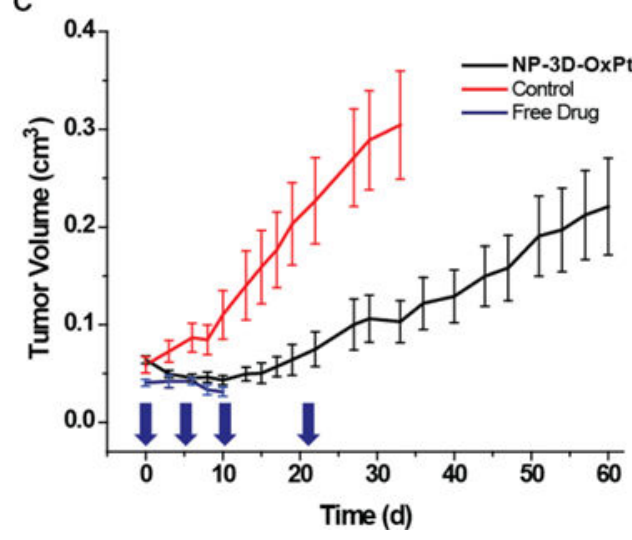

b

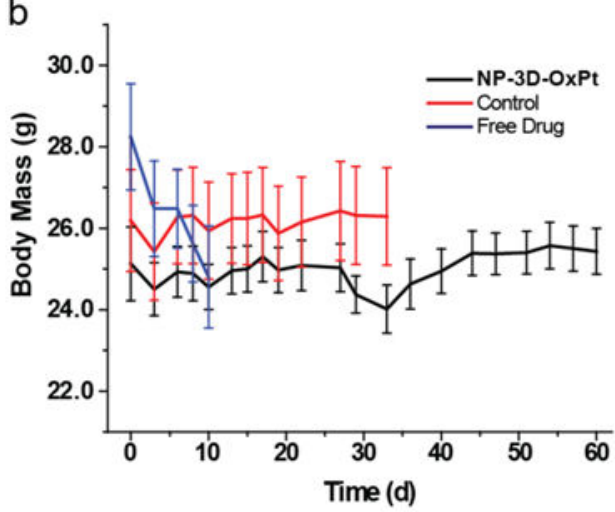

d

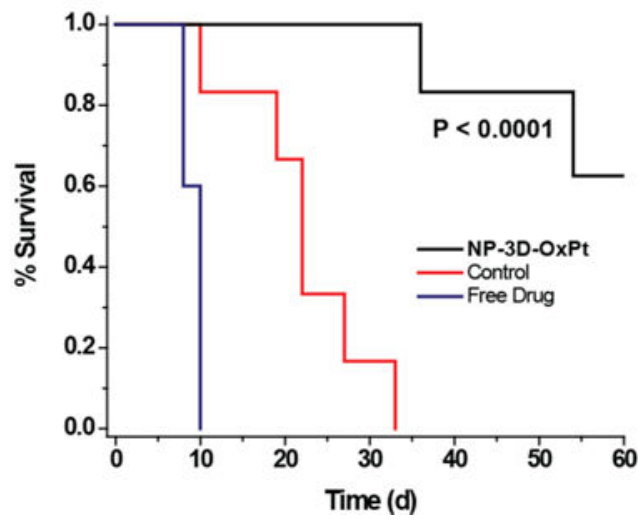

Figure 4.

Therapeutic tolerance and efficacy of a three-drug-conjugated BASP NP in comparison to its analogous free drug formulation. (a) Nanoparticle (NP) localization is observed (via epifluorescence) $20 \mathrm{~h}$ postinjection (5 mg of NP-3D-OxPt-Cy5.5)/200 $\mu \mathrm{L}$ of $5 \%$ aqueous glucose solution) in a NCR-NU mouse bearing two subcutaneous SKOV-3 xenograft tumors. Fluorescence emission $(720 \mathrm{~nm})$ was achieved through excitation $(675 \mathrm{~nm})$ of the Cy5.5 fluorophore ( $\sim 1 \%$ of NP). (b) The average body mass of the NP, control, and free drug treated groups was monitored over the course of $60 \mathrm{~d}$. Mice treated with the free drug formulation show significant (>10\%) weight loss, whereas the NP treated and vehicle control mice demonstrate consistent body masses. (c) Average tumor volume progression of the NP, control, and free drug treated groups was monitored over the course of 60 days. The treatment schedule (injections on days $0,5,11$, and 22) is marked by blue arrows just above the $x$-axis. (d) The survival (i.e., Kaplan-Meier survival curve) of the NP, control, and free drug treated groups was monitored over the course of 60 days. As a consequence of significant weight loss, the free drug treated group was euthanized before the third dose. The NP treatment group, however, finished the 60 -day study with $>60 \%$ probability of survival, while that of the vehicle control group was greatly diminished. 